\title{
Ubiquitous preservative blamed for rash of skin allergies
}

$\mathrm{T}$

he British Association of Dermatologists wants a ban on a preservative in products as diverse as face powder, paint, body wash and baby wipes, blaming it for an "epidemic" of allergic contact dermatitis.

Methylisothiazolinone is one of the most common preservatives in cosmetics and household products, but sources in Europe have found that about $1.5 \%$ of people are allergic to it (Dermatitis 2013;24:2-6). In London, UK, the rate among those tested is as high as $6 \%$. In Germany, the rate climbed from $1.9 \%$ of those tested in 2009 to $4.4 \%$ in 2011 (Contact Dermatitis 2013;68:257-8).

The rising number of people with the allergy is due to rising exposure to the substance, says American contact dermatitis expert Dr. Joe Fowler, who says he sees "quite a few" cases.

"If a potential allergen is starting to be used a lot more, and therefore you have a lot more people exposed, then you will expect to see higher incidence of allergy," he says. "That's typical."

Still, he does not think a ban is really necessary.

"When it's been done before, when they've tried to have preservatives changed or banned or whatever, some other preservative has come into the marketplace that's as bad or worse," he says. "I think it's a bit of an extreme answer."

Fowler suggests other routes, such as limiting the allowed concentration of methylisothiazolinone, putting less in products that stay on the skin and making sure that manufacturers follow the concentration rules.

Methylisothiazolinone is not a new preservative, but it used to be paired with

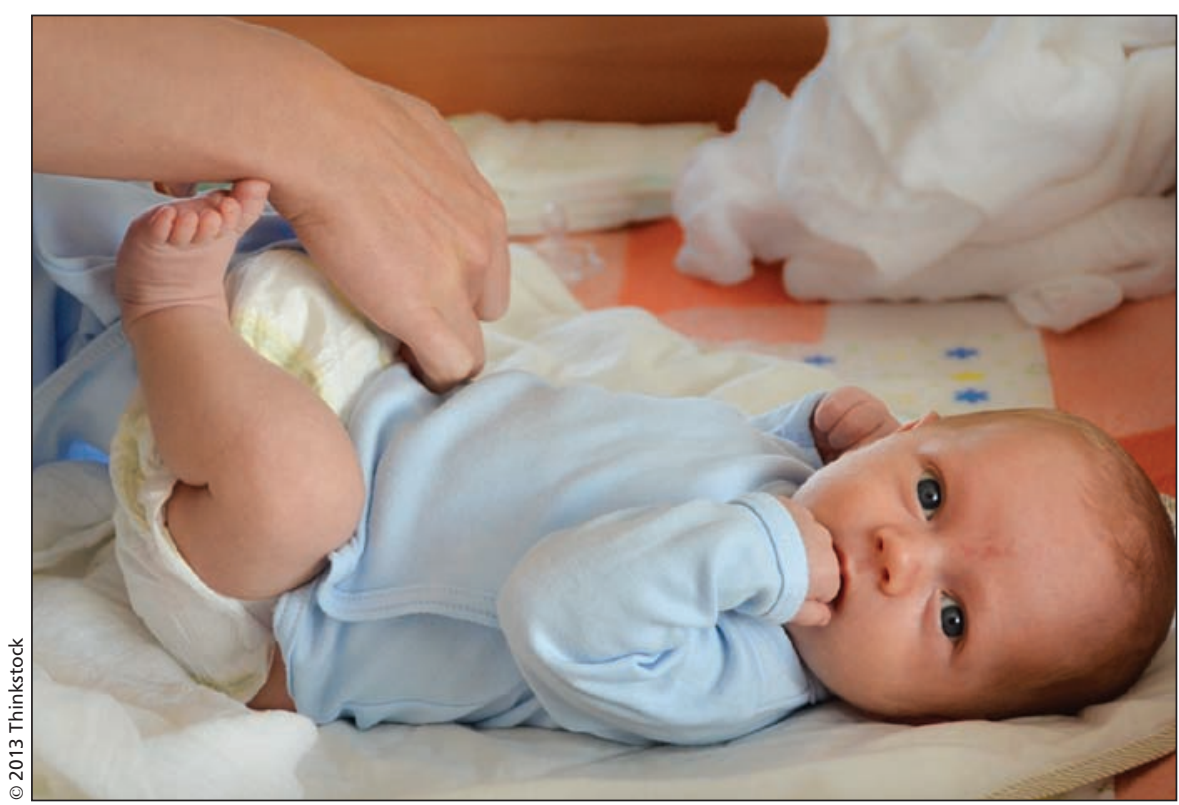

The British Association of Dermatologists wants a ban on a common preservative found in many products, including baby wipes.

methylchloroisothiazolinone. When the two were used together, methylisothiazolinone was only $25 \%$ of the mix. Based on regulatory limits, its maximum concentration in the combination was $3.75 \mathrm{ppm}$ in Canada and the United States.

Many people are allergic to the mixed preservative. However, regulators considered methylisothiazolinone a weaker allergen, which meant that, in Canada and the US, methylisothiazolinone alone was approved as a preservative for cosmetics and household products in 2005. Methylisothiazolinone is a weaker preservative on its own, so its concentration when used alone needs to be much higher than in the mix.

In many countries, including Canada and the US, methylisothiazolinone is limited to a concentration of $100 \mathrm{ppm}$. This is more than 25 times its highest legal concentration when combined with methylchloroisothiazolinone. In its higher concentration, reactions to the chemical are common. The American Contact Dermatitis Society named it their "contact allergen of the year."

The North American Contact Dermatitis Research Group, which is composed of 14 contact dermatitis experts, compiles data on common contact allergens and publishes its data in twoyear cycles. It has only recently begun to test methylisothiazolinone alone, and will be presenting the data in years to come. - Catherine Cross, CMAJ

CMAJ 2013. DOI:10.1503/cmaj.109-4570 